\title{
Properties of Conjugated Materials from Quantum Chemistry Coupled to Molecular Dynamics Generated Ensembles.
}

\author{
Andrew W. Prentice, ${ }^{\dagger}$ Jack Wildman, ${ }^{\ddagger}$ lan Galbraith, ${ }^{\ddagger}$ and Martin J. Paterson ${ }^{*, \ddagger}$ \\ $\dagger$ Department of Chemistry, University College London (UCL), London WC1H 0A, United \\ Kingdom \\ $\ddagger$ School of Engineering $\&$ Physical Sciences, Heriot-Watt University, Edinburgh EH14 4AS, \\ United Kingdom \\ E-mail: M.J.Paterson@hw.ac.uk
}




\begin{abstract}
We provide a set of molecular dynamics simulations employing a force-field specifically parameterised for organic $\pi$-conjugated materials. The resulting conformation ensemble was coupled to quantum chemistry calculations and quantities of interest for optoelectronic applications namely ground and excited state energies, oscillator strengths and dipole moments were extracted. This combined approach allowed not only exploration of the configurational landscape but also of the resulting electronic properties of each frame within the simulation and thus probe the link between conformation and property. A study was made of the sampling and convergence requirements to yield reliable averages over the ensemble. Typically between 800 and 1000 conformations were sufficient to ensure convergence of properties. However, for some oligomers more configurations were required to achieve convergence of the oscillator strength and magnitude of the dipole moment.
\end{abstract}

\title{
1. Introduction
}

The application of organic $\pi$-conjugated materials has recently been extended to the production of solar fuels, acting as photocatalysts and facilitating the reduction of $\mathrm{H}^{+}$to $\mathrm{H}_{2}$ in the presence of a sacrificial electron donor, ${ }^{1,2}$ and organic optoelectronics, such as photovoltaics ${ }^{3}$ and light emitting diodes. ${ }^{4}$ These organic systems have numerous benefits over inorganic variants, including lower device fabrication costs, as they are based on the most abundant chemical elements on earth. They allow production of lightweight devices and they are not subjected to high temperatures during processing, thereby increasing the range of potential substrates, leading to lower toxicity, chemical tunability and enhanced flexibility. ${ }^{4-8}$ Despite the advantageous chemical and mechanical properties we are yet to observe power conversion efficiencies (PCEs) in organic photovoltaics that rival their inorganic coun-

terparts. ${ }^{9-11}$ A possible source of this has attributed the to the increased disorder of organic $\pi$-conjugated materials when compared to highly ordered silicon networks. ${ }^{12}$ As these mate- 
rials have a high degree of conformational flexibility on both a single molecule level and also within the solid-state, the latter due to the presence of weak non-covalent Van der Waals interactions, insufficient transfer of charge along the polymer chain or between successive strands may occur thus limiting the overall perfomance. ${ }^{4,12}$ Additionally, the inability of the exciton to reach a donor-acceptor interface, as it is entirely possible they are located further than the typical exciton diffusion length which is between $5-10 \mathrm{~nm}$ for singlet excitons, would also contribute to the lowering of the PCE. ${ }^{12,13}$

In order to fully understand the interplay between organic $\pi$-conjugated units in a thin film and thus provide a means to explore the relationship with the demonstrated PCEs, one must first explore the structural landscape of an individual unit. A common approach to investigate the scope of potential configurations available to a dynamical system is through molecular dynamics (MD). These simulations are based upon Newton's equation of motion for which the potential energy is obtained from a force-field describing the bonded and nonbonded interactions between atoms. As the accuracy of an MD simulation is coupled to the proper description of the atomic interactions application of a such a technique to organic $\pi$-conjugated materials has been relatively hindered due to the lack of readily available optimised force-fields - these methods are primarily used in the biochemical community and are thus optimised to simulate protein dynamics. ${ }^{14-16}$ Despite readily available force-fields containing terms which would be fully transferable to organic $\pi$-conjugated materials it would be expected that re-parametrisation of various system dependent terms would have to be carried out. Previous studies by Moreno et al. ${ }^{17}$ and Bhatta et al. ${ }^{18}$ have developed force-fields for thiophene based organic $\pi$-conjugated materials, attaining various parameters from ab-initio calculations. However, as the parameterisation of the aforementioned force-fields were based on thiophene containing systems one may question the ability of these force-fields to adequately describe systems in which these moeities are not present. The discrepancy between classical force-fields and those designed directly for $\pi$-conjugtated systems was highlighted by DuBay et al. ${ }^{19}$ through comparison of inter-ring potentials of stilbenes and bithiophene 
computed from localized second order Moller-Plesset perturbation theory and the OPLS 2005 force-field, thus ensuring that prior optimisation of the potential energy terms are of utmost importance. A recent study by Wildman et al. ${ }^{20}$ sought to fill this void in available force-fields by developing a general parameterisation scheme for organic $\pi$-conjugated materials. This study built upon the general OPLS force-field ${ }^{21-27}$ for which it was found that only the intermonomer dihedral and partial charges required re-parameterisation when at larger oligomer lengths, with all other parameters adequately described within the standard OPLS force field. This study was based upon two model systems of fluorene and thiophene at varying oligomer lengths, with and without side-chains, see Fig. 1 for the general chemical structures of each. It was found that an accurate description of the intermonomer dihedral potential could be obtained by using a simple two-step geometry and energy approach employing CAM-B3LYP ${ }^{28}$ inconjuction with a $6-31 \mathrm{G}(\mathrm{d})$, and cc-pVTZ basis, respectively, when compared to $\operatorname{CCSD}(\mathrm{T}) / \mathrm{CBS}$ extrapolated energies. ${ }^{29}$ The chain length dependence was also thoroughly explored for which it was found that they had little effect on the potential energy surface of the intermonomer dihedral coordinate and could be omitted. ${ }^{20}$ The partial charge of each atom was computed using the same level of theory as mentioned previously. However, added complexity was observed for thiophene systems as one of the end side chains points towards the intermolecular chain with the other pointing outwards hereby creating an asymmetry in the charge distribution. This could be overcome by ensuring each end unit is the combination of the two appropriate units such that the non-zero charge distributions cancel one another. Despite the charges converging sufficiently when changing the oligomer length no simple way of making this process general in regards to the length of side chain was observed, with convergence of side-chains system dependent. ${ }^{20}$ The accuracy and validity of the force-field was verified from tangent-correlation functions which gave persistence lengths corresponding closely to experimental obtained values for the polymers PF8 and P3HT.

As there now exists force-fields parameterised specifically with organic $\pi$-conjugated materials in mind we now follow the next logical step and undertake MD simulations to inves- 
tigate the conformational landscape of these soft materials. In addition to this we couple the MD generated ensembles to electronic structure methods to analyse various properties including those which are relevant to optoelectronic performance. We feel that this tandem approach would ultimately aid in the design of materials with better PCEs and durability, and has been previously employed for the exploration of the absorption profile of oligothiophenes. ${ }^{30,31}$ We employ this force-field on various oligomer lengths of 9,9-dioctyl fluorene (OF8) and 3-hexyl thiophene (3HT), see Fig. 1, which have considerable interest as active electron donor materials. We provide an examination of the Kohn-Sham self-consistent field energy $(E)$, vertical ionisation energy $(I E)$, first excitation energy $\left(E^{\mathrm{S}_{1}}\right)$ and corresponding oscillator strength $(f)$, and finally the magnitude of the dipole moment $(\mu)$ across the configurational landscape, and provide a detailed statistical analysis. A comparison is also drawn between the ensemble averaged property and that obtained from a single molecule approach to explore deviations between both approaches. We hope that our analysis will guide future work as electronic structure is more often coupled with simulation of conformational landscape. We attempt to show the statistical behaviour of a common set of important molecular properties, and how the ensemble properties can differ from the traditional single minimum energy structure calculation. The effects of different sampling protocols is important to reduce computational cost while exploring a sufficient amount of the conformational space in such conjugated systems. Regardless of the specific quantum chemical model employed a smaller subset of the ensemble can produce converged properties; the degree of sampling appears to depend more on the property under consideration than the precise conjugated system. 


\section{Computational Methodology}

\subsection{Molecular Dynamics Simulations}

All MD simulations were performed through GROMACS version 4.6.5 $5^{32,33}$ and employ forcefields parameterised specifically for the specific $\pi$-conjugated material. ${ }^{20}$ Simulations were undertaken for dimer, tetramer and octamer oligomer lengths of both OF8 and 3HT, and we refer to specific oligomer lengths as an $n$ mer where $n=2,4$ or 8 . These materials were enclosed in a cubic box of chloroform and a solvent to box distance of $0.7 \mathrm{~nm}$ was used in all cases apart from the dimer systems where this was increased to $1.2 \mathrm{~nm}$ to avoid noncovalent self-interactions. We choose chloroform as a representative organic solvent which, due to its small size, would minimize the computational effort spent on simulating the solvent dynamics. The solvent density computed using our simulation methodology was also found to give an accurate representation of experimental conditions ${ }^{34}$ with respective values of $1401.6 \pm 3.9$ and $1479.5 \pm 0.5 \mathrm{~kg} \mathrm{~m}^{-3}$. The initial state of the system was generated by means of an initial steepest descent energy minimisation procedure, restricted NVT and NPT equilibration, $500 \mathrm{ps}$ of run time for each process with thermal and pressure coupling at $273.15 \mathrm{~K}$ and 1.01325 bar respectively, and lastly $10 \mathrm{~ns}$ of further NPT equilibration, now with the solute constraints lifted. The subsequent dynamics of the NPT equilibrated system was probed over $100 \mathrm{~ns}$, with solute conformations extracted every $10 \mathrm{ps}$.

\subsection{Electronic Structure Calculations}

The conformational landscapes explored in the MD simulations were processed to convert the entire solute trajectory to suitable individual input files for electronic structure analysis. As the hexyl and octyl chains are primarily utilised to aid in solubility within non-polar solvents we replace these flexible side chains with methyl groups which significantly reduces the computational cost of the quantum chemical calculations. ${ }^{35}$ For optoelectronic properties, such as the energetics and transition moment to the ${ }^{1} \pi \pi^{*}$ state, this has an almost neg- 
ligible effect. All single-point quantum chemical computations were performed exclusively in vacuo as implemented within Gaussian 09 (Revision D.01). ${ }^{36}$ For the single molecule comparison in Table 1 we use instead Gaussian 16 (Revision A.03), ${ }^{37}$ however, the discrepancy between the two versions was found to be negligible. We limit our methodology to standard density functional theory ${ }^{38,39}$ (DFT), specifically employing the range-separated hybrid CAM-B3LYP ${ }^{28}$ approximation to the exchange-correlation functional in conjunction with Dunning's polarised double zeta one-electron basis set, cc-pVDZ. In order to probe the excited state landscape we utilise the time-dependent extension to DFT (TD-DFT). ${ }^{40}$

In this initial study our overarching goal is to explore the resulting properties, and provide a statistical analysis, of a conformational space generated via specialised force-fields, and not necessarily investigating the optimal functional for comparison to relevant experimental data. Thus, various factors such as the inclusion of a solvent model, reinstatement of side-chains, greater flexibility within the one-electron basis and the inclusion of empirical dispersion corrections may all give better agreement to experiment, however, we would expect the overall statistics to remain similar to our approach.

(a)

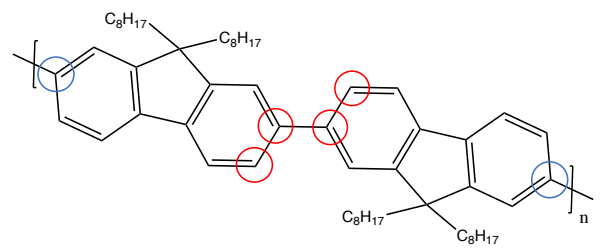

(b)

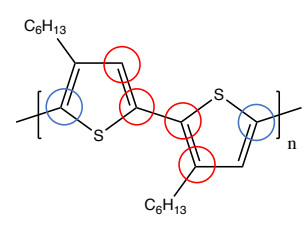

Figure 1: The molecular structure of (a) OF8, and (b) 3HT. The red and blue circles indicate the atoms used to construct the dihedral angle $(\theta)$ and the end-to-end vector, respectively. The magnitude of this vector is denoted as end-to-end length. 


\section{Results and discussion}

\subsection{Sampling Protocol}

Before reporting the statistical results we begin with a brief outline of the sampling protocol employed. As stated earlier we extract the conformation of the solute with a sampling interval $\tau_{s}=10 \mathrm{ps}$. With $100 \mathrm{~ns}$ of simulation run this yields 10001 configurations including the initial configuration. Running quantum chemistry calculations for such a large number of configurations for each molecule is an onerous task. However, it was observed that a sufficient intermonomer dihedral $(\theta)$ distribution could be obtained from a $\tau_{s}$ of 100 ps and 50 ps for OF8-2mer and 3HT-2mer respectively, see Fig. 1 for definition of this dihedral angle. We employ the general polymer naming convention for which a dihedral of $0^{\circ}$ would correspond to a trans arrangement with $180^{\circ}$ representing a cis configuration. We denote the relevant property at each point of the simulation as $x_{i}$, where $x$ is the relevant property and $i$ is the configuration number and can take any integer value in the range $1 \rightarrow 1001$ for OF8, and $1 \rightarrow 2001$ for 3HT. As shown in Fig. 2 for OF8-2mer we observe fluctuations of $\theta$ between $40^{\circ}$ and $140^{\circ}$, corresponding to the two minimum energy arrangements along the $\theta$ coordinate. These minima are essentially isoenergetic as they only differ by $0.11 \mathrm{~kJ}$ $\mathrm{mol}^{-1}$, and are connected via a high energy transition state at $90^{\circ}$ which has a height of 9.0 $\mathrm{kJ} \mathrm{mol}^{-1}$. Therefore, based on a Boltzmann population analysis we would expect to find roughly $51.1 \%$ of the $\theta$ values across the ensemble to be below $90^{\circ}$, and the other $48.9 \%$ to be above. For OF8-2mer we find good agreement to this with a split of $52 \%$ of $\theta<90^{\circ}$ and $48 \%$ of $\theta>90^{\circ}$. As the simulations were performed at $273.15 \mathrm{~K}$, resulting in a $N_{A} k T$ of 2,271 $\mathrm{kJ} \mathrm{mol}^{-1}$, and from the relative steepness of the surface around the minimum arrangements we would expect little occupation of $\theta$ outwith this range, which is observed. However, in the case of 3HT-2mer we observe a far more spread-out set of $\theta$ values. Upon inspection of the potential energy surface along the $\theta$ coordinate we once again observe isoenergetic minima at around $40^{\circ}$ and $140^{\circ}$. The barrier connecting the minima is far lower in the case of $3 \mathrm{HT}-2 \mathrm{mer}$ 
with a value of $3.9 \mathrm{~kJ} \mathrm{~mol}^{-1}$ and the surface around these geometries is much flatter leading to a more spread out set of values. Upon examination of the spread of $\theta$ below and above $90^{\circ}$ we now observe a ratio of $68: 32 \%$. This discrepancy could be attributed to the more asymmetrical nature of the surface and thus it would be more probable for a configuration with a dihedral at $40^{\circ}$ to interchange to a symmetrical configuration located at $-40^{\circ}$. In the case of the larger dihedral minimum the barriers to the symmetric configuration and the trans arrangement are much more alike.

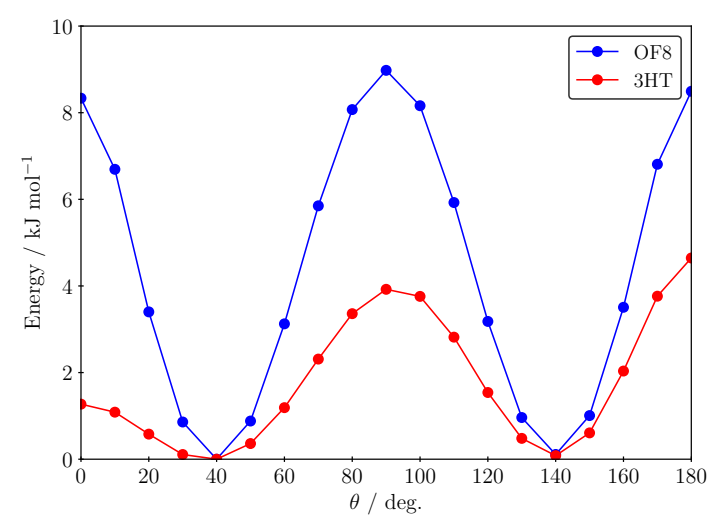

(a)

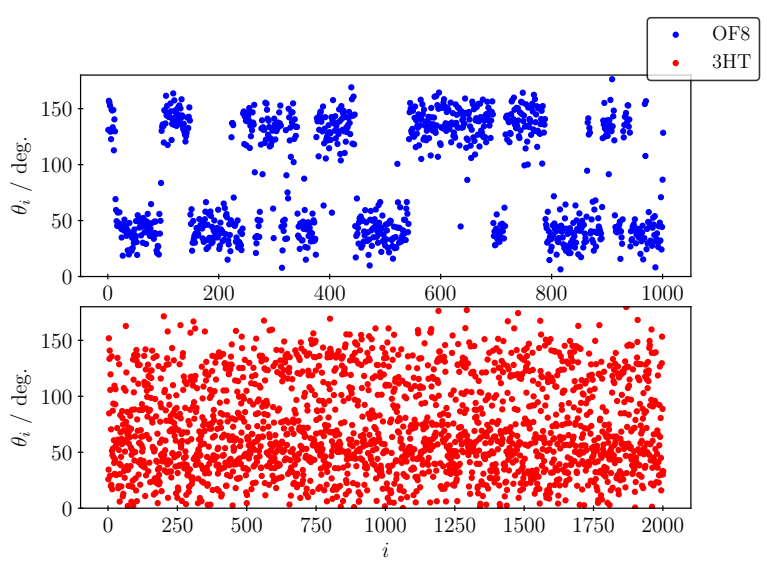

(b)

Figure 2: (a) Potential energy surface corresponding to the intermolecular dihedral angle for OF8 and 3HT-2mer. (b) The intermolecular dihedral for each individual OF8 and 3HT-2mer molecule within the simulated trajectory.

In addition to the convergence of the dihedral distribution within these reduced ensembles we also investigated the auto-correlation function $(\alpha)$, as outlined in Eq. 1 for the specific case of a $\tau_{s}$ of 50 and 100 ps for OF8 and 3HT respectively, where $\bar{x}$ is the ensemble average and $n$ is the number of individual frames within the ensemble.

$$
\alpha\left(\tau_{s}\right)=\frac{\sum_{i=1}^{n-1} P_{i} P_{i+1}}{\sum_{i=1}^{n} P_{i}^{2}} ; P_{i}=x_{i}-\bar{x}
$$

This function gives an indication of the degree of correlation between adjacent conformations, which in the limit of $\tau_{s}$ approaching $+\infty$ is 0 thus representing truly uncorrelated samples. 
Practically the property is said to be uncorrelated at a specific time-step if $\alpha \leq e^{-1} \approx$ 0.37. A compromise between over and under-sampling a simulation must be maintained as simulations must contain enough suitable uncorrelated points whilst still remaining in the realms of computational feasibility by employing realistic time-steps.

For OF8 and 3HT-2mer the auto-correlation was investigated for the energy, deviation from molecular plane $(\phi)$ and end-to-end length $(R)$. The planar deviation quantity involved scaling all the dihedral angles that were greater than $90^{\circ}$ by $180^{\circ}-\theta$, and $R$ is the standard Euclidean distance between the furthest two carbon atoms in a straight chain linear polymer, as defined by the blue circles in Fig. 1 for both polymers. In all instances we find uncorrelated properties for this specific time-step. We also extended this check to the largest oligomers considered within this study for which we find that the energy is uncorrelated. However, for $R$ we instead find a slight correlation at a $\tau_{s}$ of 100 and $50 \mathrm{ps}$, with values of 0.63 and 0.65 for OF8 and 3HT-8mer respectively.

\subsection{Electronic Energy}

We begin with perhaps the most fundamental property of the system, the ground state electronic energy as obtained via self-consistent solution of the Kohn-Sham equations. ${ }^{38,39}$ For the ensemble statistics we use the population definitions, as implemented within the

SciPy open source software package, ${ }^{41}$ for the $\bar{x}$, standard deviation $\left(x_{\sigma}\right)$, skewness $\left(x_{\gamma}\right)$ and kurtosis $\left(x_{\beta}\right)$. For completeness we provide all ensemble statistics in Table 1 for all the oligomers and properties of interest. The skewness and kurtosis are also referred to as the third and fourth moment of the distribution and quantify the deviations from a normal unimodal data set, for which both these quantities would equal zero. The former provides information regarding the degree of asymmetry within the data set and the latter indicates the quantity of outliers, both of which are relative to a normal unimodal distribution..

The electronic energy for each individual configuration within the OF8 and 3HT ensembles are provided in Fig. 3(a), with the larger oligomers provided in Fig. S1, we provide these 
Table 1: Overall ensemble statistics for all systems and properties investigated. The two rightmost columns give to the property at a single optimised anticonformation $\left(x_{S M}\right)$ and the corresponding difference $(\Delta x)$ with respect to the average $\bar{x}$.

\begin{tabular}{|c|c|c|c|c|c|c|c|}
\hline Oligomer & Range (eV) & $\bar{E}$ (hartree) & $E_{\sigma}(\mathrm{eV})$ & $E_{\gamma}(\mathrm{eV})$ & $E_{\beta}(\mathrm{eV})$ & $E_{S M}$ (hartree) & $\Delta E(\mathrm{eV})$ \\
\hline OF8-2mer & 1.133 & -1158.22940 & 0.181 & 0.315 & 0.126 & -1158.28176 & 1.425 \\
\hline OF8-4mer & 1.776 & -2315.27638 & 0.263 & 0.240 & 0.051 & -2315.38007 & 2.822 \\
\hline OF8-8mer & 2.724 & -4629.36891 & 0.374 & 0.187 & 0.322 & -4629.57668 & 5.654 \\
\hline $3 \mathrm{HT}-2 \mathrm{mer}$ & 0.973 & -1183.24089 & 0.133 & 0.472 & 0.392 & -1183.26220 & 0.580 \\
\hline $3 \mathrm{HT}-4 \mathrm{mer}$ & 1.268 & -2365.29825 & 0.185 & 0.350 & 0.093 & -2365.34175 & 1.184 \\
\hline $3 \mathrm{HT}-8 \mathrm{mer}$ & 1.952 & -4729.11132 & 0.273 & 0.198 & -0.055 & -4729.50101 & 10.604 \\
\hline Oligomer & Range (eV) & $\overline{I E}(\mathrm{eV})$ & $I E_{\sigma}(\mathrm{eV})$ & $I E_{\gamma}(\mathrm{eV})$ & $I E_{\beta}(\mathrm{eV})$ & $I E_{S M}(\mathrm{eV})$ & $\Delta I E(\mathrm{eV})$ \\
\hline OF8-2mer & 0.734 & 7.087 & 0.098 & 1.131 & 2.444 & 7.085 & 0.002 \\
\hline OF8-4mer & 0.601 & 6.766 & 0.077 & 0.513 & 0.729 & 6.768 & -0.002 \\
\hline OF8-8mer & 0.400 & 6.632 & 0.052 & 0.260 & 0.569 & 6.643 & -0.011 \\
\hline $3 \mathrm{HT}-2 \mathrm{mer}$ & 1.537 & 7.704 & 0.288 & 1.195 & 1.473 & 7.522 & 0.182 \\
\hline $3 \mathrm{HT}-4 \mathrm{mer}$ & 1.650 & 7.066 & 0.226 & 0.642 & 0.994 & 6.660 & 0.406 \\
\hline $3 \mathrm{HT}-8 \mathrm{mer}$ & 1.069 & 6.785 & 0.176 & 0.145 & -0.355 & 6.225 & 0.560 \\
\hline Oligomer & Range (eV) & $\bar{E}^{\mathrm{S}_{1}}(\mathrm{eV})$ & $E_{\sigma}^{\mathrm{S}_{1}}(\mathrm{eV})$ & $E_{\gamma}^{\mathrm{S}_{1}}(\mathrm{eV})$ & $E_{\beta}^{\mathrm{S}_{1}}(\mathrm{eV})$ & $E_{S M}^{\mathrm{S}_{1}}(\mathrm{eV})$ & $\Delta E^{\mathrm{S}_{1}}(\mathrm{eV})$ \\
\hline OF8-2mer & 0.791 & 4.220 & 0.134 & 0.731 & 0.681 & 4.227 & -0.007 \\
\hline OF8-4mer & 0.800 & 3.849 & 0.105 & 0.501 & 0.629 & 3.854 & -0.005 \\
\hline OF8-8mer & 0.613 & 3.689 & 0.079 & 0.352 & 0.491 & 3.708 & -0.019 \\
\hline $3 \mathrm{HT}-2 \mathrm{mer}$ & 1.509 & 4.768 & 0.327 & 0.342 & -0.740 & 4.593 & 0.175 \\
\hline $3 \mathrm{HT}-4 \mathrm{mer}$ & 1.841 & 4.091 & 0.294 & 0.394 & 0.054 & 3.590 & 0.501 \\
\hline 3HT-8mer & 1.330 & 3.752 & 0.229 & 0.170 & -0.289 & 3.059 & 0.693 \\
\hline Oligomer & Range & $\bar{f}$ & $f_{\sigma}$ & $f_{\gamma}$ & $f_{\beta}$ & $f_{S M}$ & $\Delta f$ \\
\hline OF8-2mer & 0.409 & 1.350 & 0.059 & -0.879 & 1.402 & 1.412 & -0.062 \\
\hline OF8-4mer & 1.152 & 3.187 & 0.204 & -0.421 & -0.201 & 3.497 & -0.310 \\
\hline OF8-8mer & 4.318 & 6.065 & 0.760 & -0.304 & -0.105 & 7.400 & -1.335 \\
\hline $3 \mathrm{HT}-2 \mathrm{mer}$ & 0.443 & 0.304 & 0.084 & -1.453 & 2.227 & 0.379 & -0.075 \\
\hline $3 \mathrm{HT}-4 \mathrm{mer}$ & 0.764 & 0.874 & 0.110 & -0.190 & -0.104 & 1.124 & -0.250 \\
\hline 3HT-8mer & 1.850 & 1.710 & 0.311 & 0.045 & -0.212 & 2.677 & -0.967 \\
\hline Oligomer & Range (D) & $\bar{\mu}(\mathrm{D})$ & $\mu_{\sigma}(\mathrm{D})$ & $\mu_{\gamma}(\mathrm{D})$ & $\mu_{\beta}(\mathrm{D})$ & $\mu_{S M}(\mathrm{D})$ & $\Delta \mu(\mathrm{D})$ \\
\hline OF8-2mer & 1.355 & 0.690 & 0.299 & -0.178 & -0.980 & 0.336 & 1.019 \\
\hline OF8-4mer & 2.049 & 0.984 & 0.425 & 0.221 & -0.511 & 0.700 & 1.349 \\
\hline OF8-8mer & 2.293 & 1.127 & 0.475 & 0.393 & -0.352 & 1.381 & 0.912 \\
\hline $3 \mathrm{HT}-2 \mathrm{mer}$ & 1.871 & 0.956 & 0.431 & -0.041 & -1.090 & 0.564 & 1.307 \\
\hline $3 \mathrm{HT}-4 \mathrm{mer}$ & 3.003 & 1.303 & 0.516 & 0.237 & -0.329 & 0.930 & 2.073 \\
\hline $3 \mathrm{HT}-8 \mathrm{mer}$ & 3.922 & 1.742 & 0.641 & 0.223 & -0.249 & 1.506 & 2.416 \\
\hline
\end{tabular}


energies relative to $\bar{E}$, however, all statistical measurements will remain unchanged. For all systems we obtain a fairly equilibrated spread of energies oscillating above and below $\bar{E}$. As highlighted in Table 1 the range of energies encountered and the ensemble deviation with respect to the single molecule picture both increase as a function of $n$. This is expected as the magnitude of the energies and the number of degrees of freedom both increase, and as the energy is essentially a sum of contributions from each atom we would expect larger fluctuations for the longer oligomers. The standard deviation also follows this logic increasing from 0.181 $\mathrm{eV}$ to $0.374 \mathrm{eV}$ and from $0.133 \mathrm{eV}$ to $0.274 \mathrm{eV}$ across $\mathrm{OF} 8$ and $3 \mathrm{HT}$ oligomers, respectively. As illustrated for all oligomers in Fig. 3(b), we observe that each data set is very similar to a normal unimodal distribution, computed from the associated ensemble mean and standard deviation, further highlighted by the small skewness and kurtosis values of these data sets for this particular property. The number of configurations needed to observe convergence
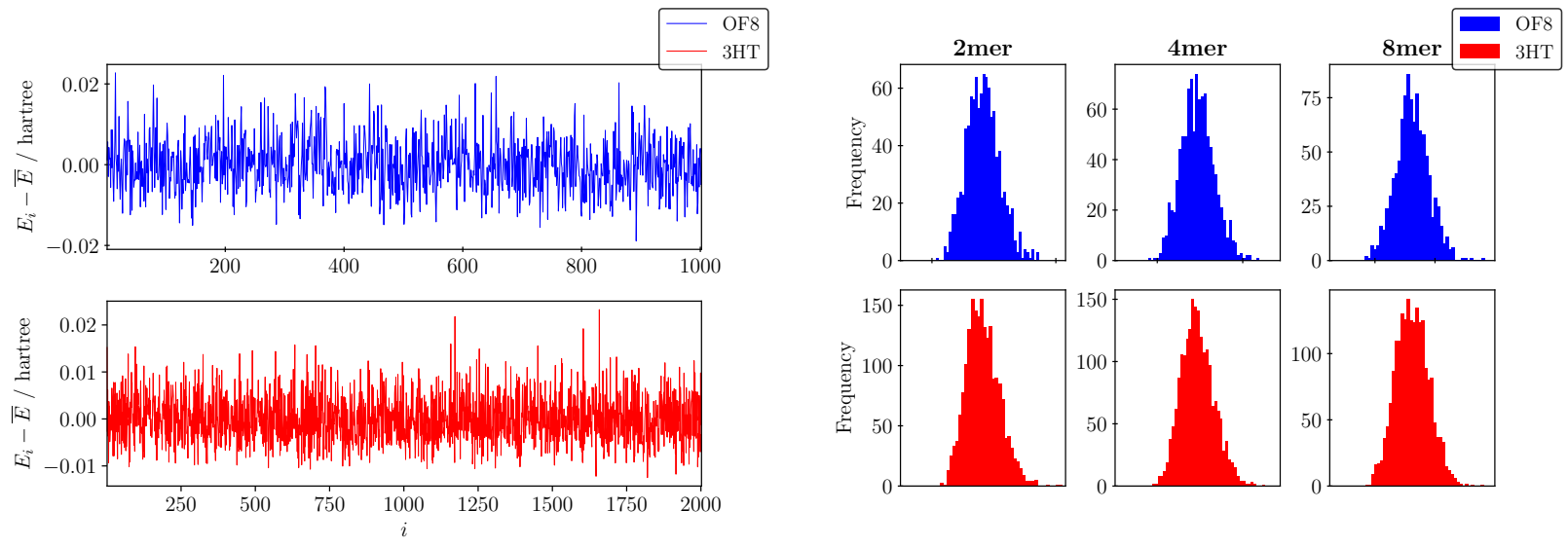

(a)

(b)

Figure 3: (a) $E_{i}-\bar{E}$ for each individual OF8 and 3HT-2mer molecule within the simulated trajectory. (b) Histogram representation of the electronic energy for the ensembles considered, the abscissa of the histogram spans $\pm 5 E_{\sigma}$ from $\bar{E}$.

to the ensemble mean was then explored using the relative running average of the deviation from the whole ensemble mean as defined in Eq. 2,

$$
\widetilde{x}_{j}=\frac{1}{\bar{x}} \frac{1}{j} \sum_{i=1}^{j}\left(x_{i}-\bar{x}\right)
$$


where $j$ runs up to 1001 for OF8 and to 2001 for 3HT. This allows us to investigate how many successive configurations are required to give a sufficient representation of the ensemble averaged value. By definition the value of $\widetilde{x}_{j}$ for the largest $j$ will equal zero.

As shown in Fig. 4 for all oligomer lengths we start to observe convergence at around 800 configurations. It is clear that going above 1000 configurations for 3HT has no effect on the energy convergence. However, the magnitude of the scale should be noted as the upper and lower limits correspond to a difference of only $\pm 1 \times 10^{-4} \%$ and $\pm 5 \times 10^{-5} \%$ for $\mathrm{OF} 8$ and $3 \mathrm{HT}$, respectively. Therefore, to assess the convergence of the moving average more quantitatively we have added lines corresponding to $10 \%$ of the relevant standard deviation. For both sets of molecules it is clear that the ensemble averaged energy can be well approximated within this energy tolerance with far reduced subsets of the overall trajectory. For OF8-4mer this is achieved when using subsets that contain less than one fifth of the original number contained within the ensemble. In terms of OF8-2mer and OF8-8mer this is larger at approximately 400-500 configurations. For the 3HT oligomers all values are within these reduced limits by 400 configurations, with the 4 mer and 8 mer essentially well approximated outwith the initial fluctuation at the start of the moving average. Note this conclusion depends on having properly sampled ensembles as discussed in Section 3.1. 

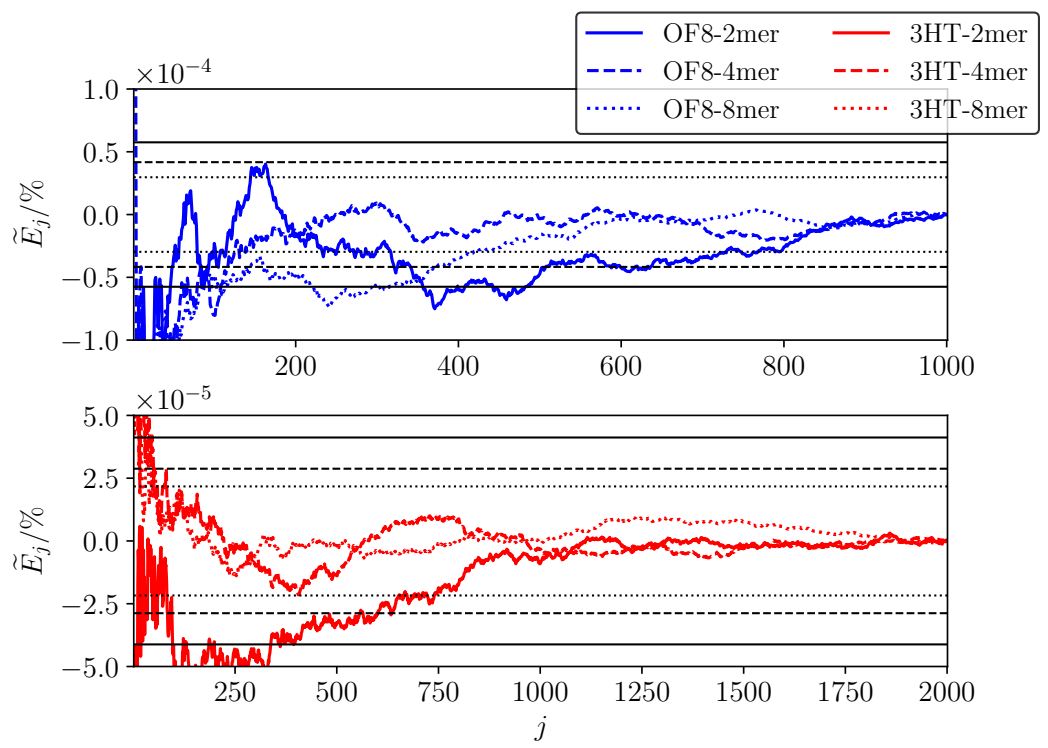

Figure 4: Convergence of $\widetilde{E}_{j}$ for each successive point across the simulated trajectory. The horizontal lines represent a deviation of $\pm \frac{1}{10} E_{\sigma}$ either side of the average $\bar{E}$.

\subsection{Ionisation Energy}

The next property we investigated was the vertical ionisation energy $(I E)$ of the aforementioned ensembles, due to its importance for processes such as electron transfer. We investigate the vertical ionisation energy which is the energy difference between the neutral and cationic state with the same conformation. The ionisation energy for the configurational landscape of $\mathrm{OF} 8$ and 3HT once again provide an equilibrated spread of data points as previously observed for the electronic energy, see Fig. S2. From the ensemble statistics the IE range was found to decrease in a step-wise fashion for the OF8 systems only, with respective values of $0.734 \mathrm{eV}, 0.601 \mathrm{eV}$ and $0.400 \mathrm{eV}$ for increasing oligomer lengths (See Table 1). This was not the case for $3 \mathrm{HT}$ systems as the range increased and finally decreased by $0.113 \mathrm{eV}$ and $0.581 \mathrm{eV}$ for successive increasing oligomer lengths. For both materials the average $\overline{I E}$ was found to obey this step-wise diminishing behaviour which would be expected as the $\pi$-conjugation of the molecule increases. When we compare the single molecule approach with the ensemble averaged value we find for OF8 oligomers these quantities are essentially identical, deviating at most by $0.011 \mathrm{eV}$ for the 8 mer. In terms of $3 \mathrm{HT}$ we observe a larger 
discrepancy between the two approaches with deviations of $0.182 \mathrm{eV}, 0.406 \mathrm{eV}$ and $0.560 \mathrm{eV}$ for increasing oligomer lengths. As determination of the ionisation potential involves the ground state energy the statistics previously outlined for the energy will be inherently included.

As outlined in Fig. 5(a) as we increase the oligomer lengths the likeness to a unimodal normal distribution strengthens. For OF8-2mer and 3HT-2mer we observe distributions that are positively skewed to a considerable extent which can be clearly observed in the relevant histograms, and also by the kurtosis and skewness values in Table 1. For 3HT2 mer we observe a small grouping of data points centred at $\sim 8.5 \mathrm{eV}$ generating a bimodal distribution. This smaller peak can be attributed to the cluster of larger $I E$ values centred around a $\theta$ of $90^{\circ}$, see Fig. 5(b).

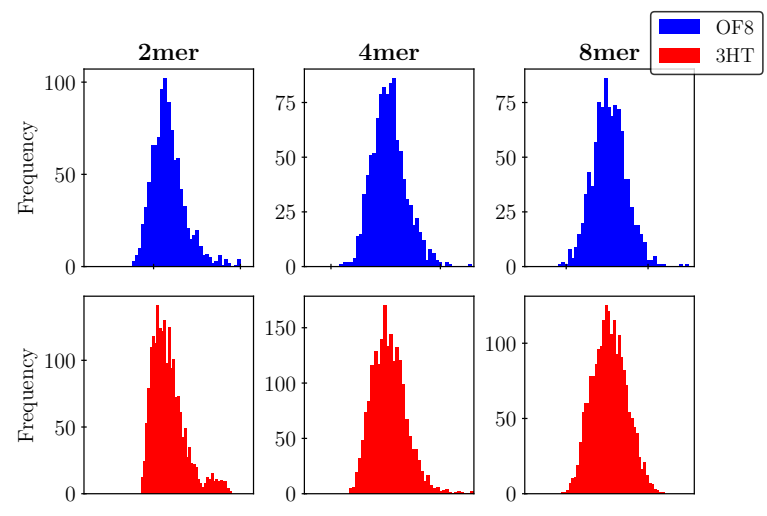

(a)

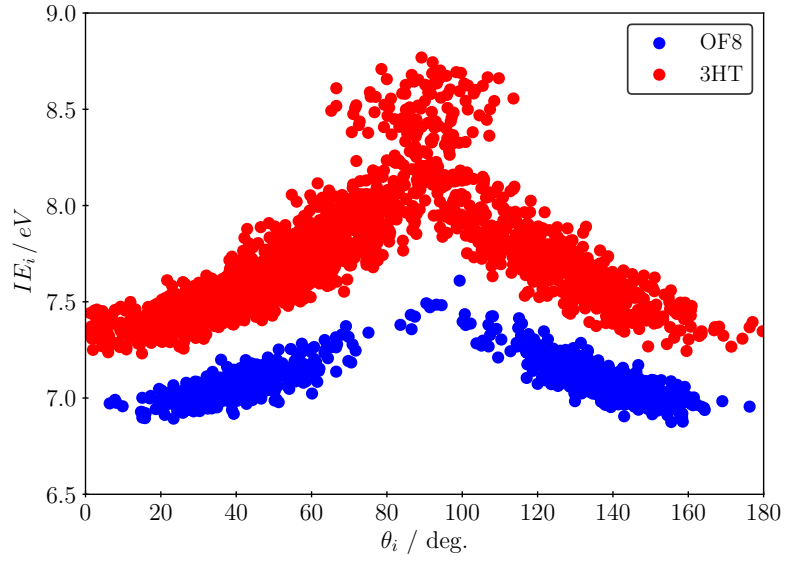

(b)

Figure 5: (a) Histogram representation of the ionisation for the ensembles considered, the abscissa of the histogram data spans $\pm 5 I E_{\sigma}$ from $\overline{I E}$. (b) The ionisation energy as a function of the intermolecular dihedral for OF8 and 3HT-2mer ensembles.

The convergence to the ensemble average was then explored in a similar fashion to the previous section, see Fig. S3. All the OF8 oligomers show convergence by 800 configurations, and a slightly higher configuration amount of 1000 configurations is sufficient for 3HT oligomers. However, for the 8 mer ensembles we observe a convergence at a far earlier point 
within the simulation, approximately 400 and 750 configurations for OF8 and 3HT respectively. In terms of the increased tolerance limits for the OF8-2mer and 8mer systems we observed this crossing at around 275 and 200 configurations, respectively, with the OF84mer requiring more configurations to reach these limits. A similar finding is observed for the corresponding $3 \mathrm{HT}$ oligomers.

\subsection{First Excited State}

We now turn our attention to the first excited state energy $\left(E^{\mathrm{S}_{1}}\right)$ and oscillator strength $(f)$ of each configuration as computed via TD-DFT. As these molecules are commonly employed as electron donors after photoexcitation, knowledge of this excited state is important. We limit the excited state portion to the first vertical excited state of each configuration following the standard Kasha's rule approach. The raw data pertaining to the 2 mer systems are provided in Fig. 6(a) and Fig. 6(b) for $E^{\mathrm{S}_{1}}$ and $f$, respectively, see Fig. S4 and Fig. S5 for the relevant data for the longer oligomers. In addition we explored the angle between the end-to-end vector, as defined in Fig. 1, and the transition dipole moment vector for this state $\left(\Theta^{T}\right)$ for the largest and smallest oligomers of each system. As shown in Fig. S6 we observe $\Theta$ values of $\sim 0$ or $\sim 180^{\circ}$, therefore highlighting that these vectors are practically parallel for each configuration. This makes sense as the lowest excited state for these systems is of $\pi \pi^{*}$ character localised along the molecular backbone. Once again upon increasing the conjugation length the ensemble mean energy is found to decrease, from $4.22 \mathrm{eV}$ to $3.69 \mathrm{eV}$ for $\mathrm{OF} 8-2 \mathrm{mer}$ to $\mathrm{OF} 8-8 \mathrm{mer}$, and from $4.77 \mathrm{eV}$ to $3.75 \mathrm{eV}$ in the case of the $3 \mathrm{HT}$ counterpart (see Table 1). The oscillator strength is found to increase almost linearly with the increasing oligomer length. In most instances for the organic conjugated oligomers the lowest energy transition is dominated by a single particle-hole transition involving a $\pi \pi^{*}$ transition localised along the molecular backbone. As we increase the oligomer length the excited state involves a number of particle-hole transitions of $\pi \pi^{*}$ character and localised at various points over the conjugated backbone. For $E^{\mathrm{S}_{1}}$ we observe equilibrated spread of data points for all 
systems considered. In terms of the difference with respect to a single quantum mechanical calculation for $E_{i}^{\mathrm{S}_{1}}$ we see a similar behaviour to the ionisation energy. In all cases the OF8 ensemble averaged value is practically the same between these two approaches, however, we observe non-negligible differences for the 3HT oligomers. In terms of the oscillator strength of the first excited state each configuration within the ensemble generated an optically bright state, with the exception of 3HT-2mer which oscillates between a bright and dark state. The difference with respect to the single molecules is also apparent for not only $3 \mathrm{HT}$ oligomers but for OF8, and increases for successive lengths. As was true with the $I E$ an almost symmetric plot is observed when investigating $E^{\mathrm{S}_{1}}$ and $f$ as a function of intermolecular dihedral, see Fig. 6(c) and Fig. 6(d). The dark states for 3HT-2mer can be attributed to the small grouping of configurations with an intermolecular dihedral of approximately $90^{\circ}$, however, for the OF8-2mer counterparts these are still considerably bright.

As observed in Table 1 and Fig. 7(a) we once again observe a better agreement to a normal unimodal distribution upon increasing the conjugated backbone length, however, we do not observe any apparent difference between 3HT-4mer and 3HT-8mer. When we observe the relevant statistics for $f, f_{\sigma}$ increases for each successive oligomer for both systems, in contrast to $E^{\mathrm{S}_{1}}$, with $f_{\gamma}$ and $f_{\beta}$ values decreasing. For $3 \mathrm{HT}-2 \mathrm{mer}$ statistics the effect of the small grouping resulting in a clear negative skewed distribution for $f$ and a flatter distribution for $E^{\mathrm{S}_{1}}$. The negative skewness in terms of $f$ for PF8-2mer is also apparent from the relevant histogram in Fig. 7(b).

We then examined the moving average for both excited state properties, see Fig. 8(a) and Fig. $8(\mathrm{~b})$ for $E^{\mathrm{S}_{1}}$ and $f$, respectively. In terms of $E^{\mathrm{S}_{1}}$ for OF8 we observe convergence for all oligomer lengths at approximately 800 configurations, which for thiophene was found to be more at 1100 configurations. Once again, the largest oligomers show this converging behaviour at far earlier points within the simulation. When we look at the increased tolerance range OF8-8mer converges for all moving averages outwith the initial fluctuation. For OF82 mer this occurs under 200 configurations, however, for the 4 mer this is larger at around 

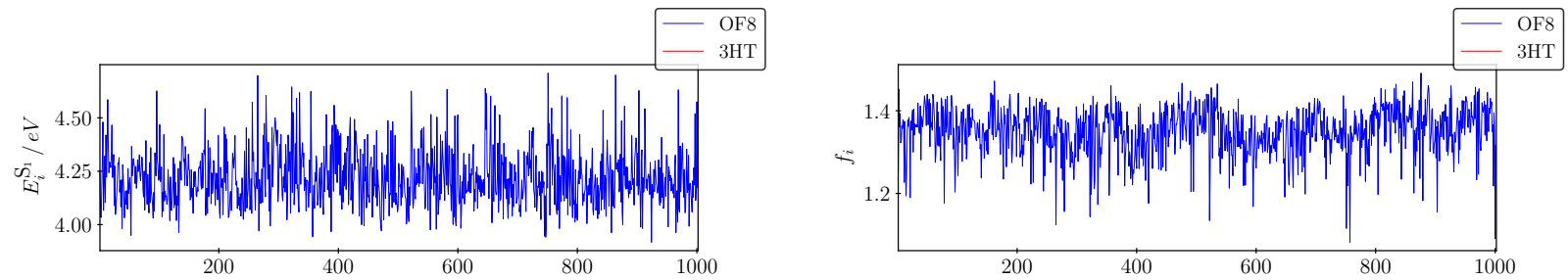

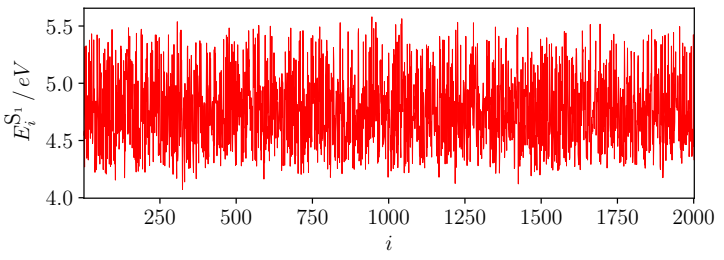

(a)

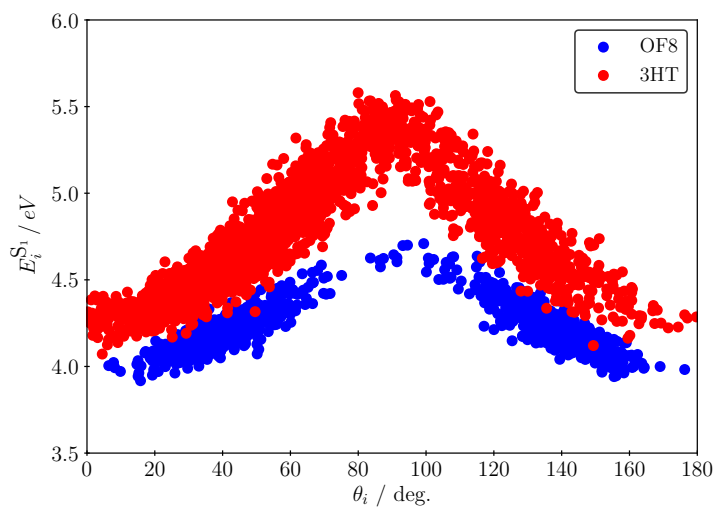

(c)

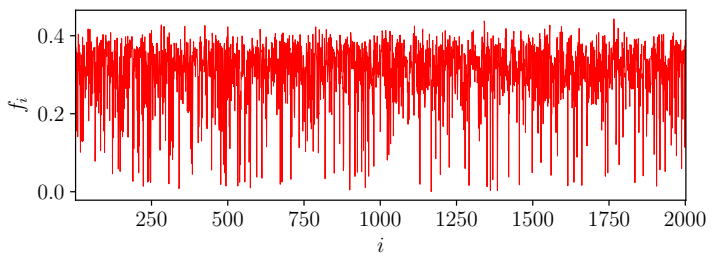

(b)

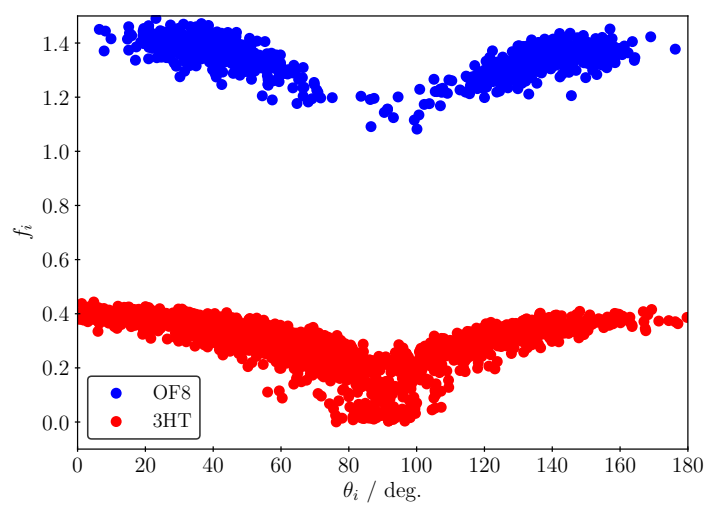

(d)

Figure 6: (a) The first excitation energy for each individual OF8 and 3HT-2mer molecule within the simulated trajectory. (b) The oscillator strength of the first excited state for each individual $\mathrm{OF} 8$ and $3 \mathrm{HT}-2$ mer molecule within the simulated trajectory. (c) The first excitation energy as a function of the intermolecular dihedral for each individual OF8 and 3HT-2mer molecule within the simulated trajectory. (d) The oscillator strength of the first excited state as a function of the intermolecular dihedral for each individual OF8 and 3HT2mer molecule within the simulated trajectory. 

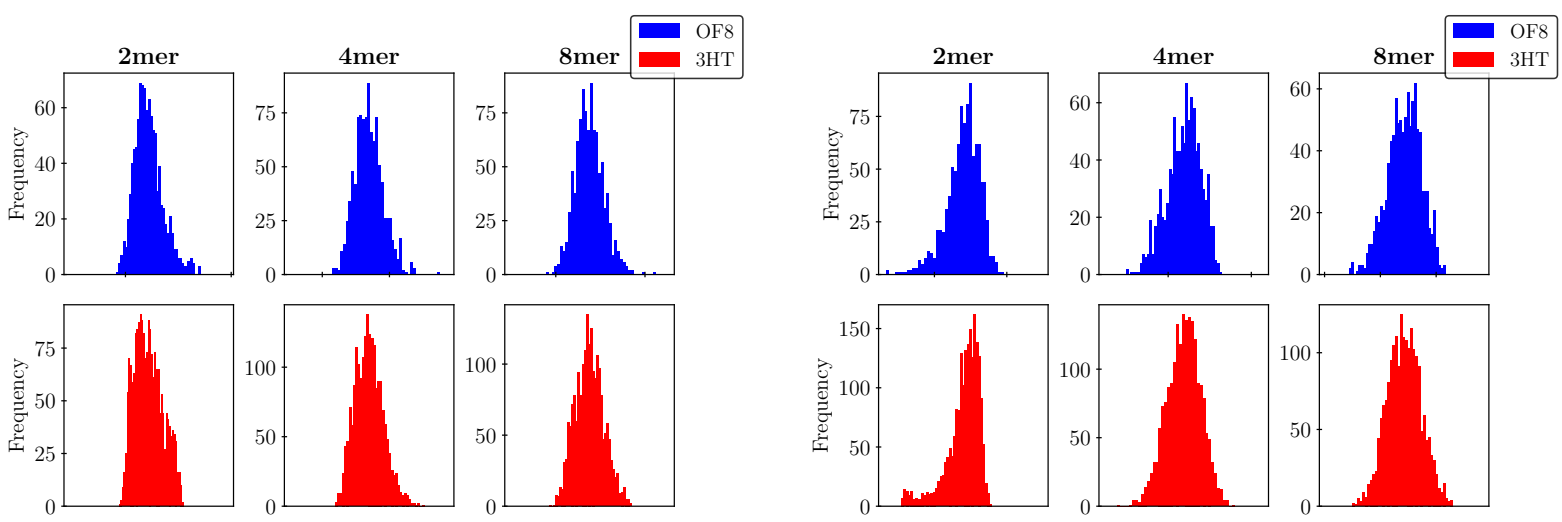

(a)
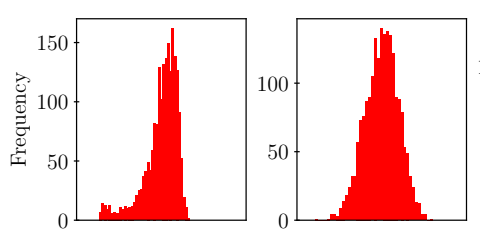

(b)

Figure 7: Histogram representation of (a) the first excitation energy for the ensembles considered, the abscissa of the histogram spans $\pm 6 E_{\sigma}^{S_{1}}$ from $\bar{E}_{i}^{S_{1}}$. (b) the oscillator strength of the first excited state for the ensembles considered, the abscissa of the histogram spans $\pm 5 f_{\sigma}$ from $\bar{f}$.

500 configurations. For $3 \mathrm{HT}$ the $2 \mathrm{mer}$ is within these limits for all configurations with the 4 mer and 8mer within these limits at 500 configurations. Once again this shows that the ensemble averaged value can be reached in subsets containing far fewer configurations. We do not observe as good as a convergence with respect to the oscillator strength. For OF8 systems we observe fluctuations across the entire set of moving averages, with the OF8-2mer and 8 mer showing converge around 900 configurations. For $3 \mathrm{HT}$ we observe a similar story with the start of convergence reached around 1500 configurations 3HT-2mer and 3HT-4mer. The 3HT-8mer does not reach convergence for any reduced subset. It could be argued that the OF8-2mer and 3HT-4mer reach convergence earlier, however, the convergence is not as clear as the previous energetic based properties and strong oscillations are still observed until later stages of the simulation. In terms of the increased tolerance range for OF8-2mer and OF8-8mer we find this crossing point at around 275 and 350 configurations, respectively. For the 4 mer far more configurations are required, upwards of 900 to reach this limit. This is in contrast to the $3 \mathrm{HT}$ systems for which the 4 mer has reached this limit by subsets containing only 250 configurations, which is also true for the 3 HT-2mer. It is the 8 mer that 
requires far more configurations, reaching this reduced energy range at approximately 1700 configurations.
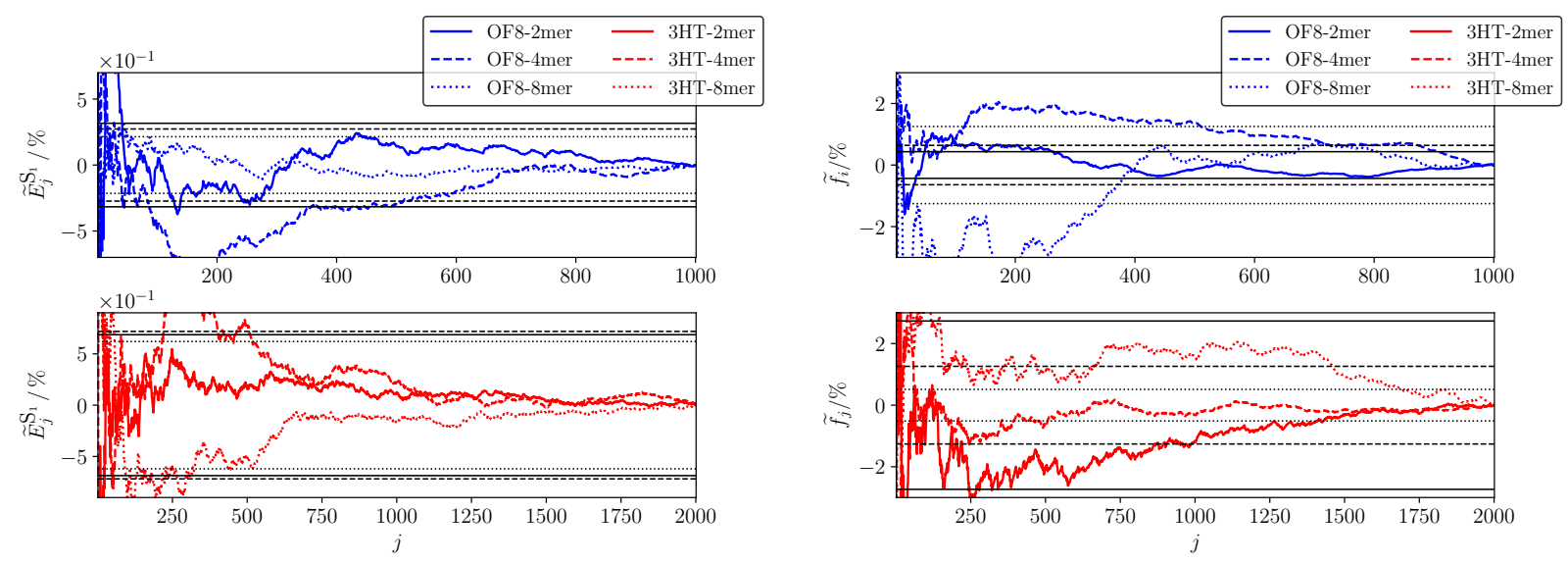

(a)

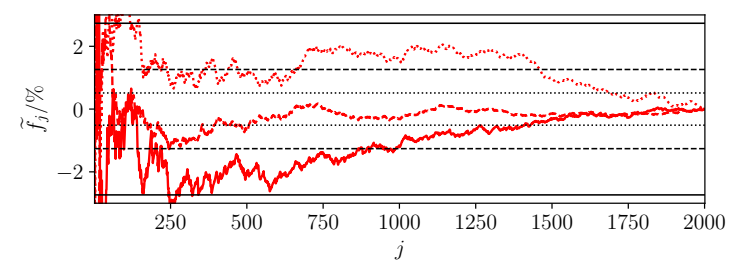

(b)

Figure 8: Convergence of (a) $\widetilde{E}_{j}^{\mathrm{S}_{1}}$ for each successive point across the simulated trajectory. The solid lines represent the quantity which would represent a deviation of $\pm \frac{1}{10} E_{\sigma}^{\mathrm{S}_{1}}$ from $\bar{E}^{\mathrm{S}_{1}}$ (b) $\tilde{f}_{i}$ for each successive point across the simulated trajectory. The solid lines represent the quantity which would represent a deviation of $\pm \frac{1}{10} f_{\sigma}$ from $\bar{f}$.

\subsection{Magnitude of the Dipole Moment}

We now turn our attention to a non-energetic property of the ensembles, specifically the magnitude of the dipole moment. It is clear from the plot of the raw data for OF8-2mer we do not observe the same equilibrated spread of values as obtained previously, see Fig. 9(a) for the 2mers and Fig. S7 for the other ensembles. Instead the property equilibrates and then a considerable fluctuation occurs, once again establishing an equilibrium and continuing within this regime, this is in a similar fashion to the $\theta$ distribution for the OF8-2mer. When we investigate the magnitude of the dipole moment as a function of intermolecular dihedral we do not observe the symmetrical behaviour observed for the previous properties. For OF8-2mer we observe a grouping of points with a lower dipole magnitude with another grouping at larger dipole moments centred around the two minimum energy arrangements, see Fig.9(b). For 3HT-2mer we instead find a roughly linear relationship between the inter- 
molecular dihedral and the dipole moment, see Fig.9(c). This can be clearly observed in the histograms as illustrated in Fig 9(d) for which we observe a clear bimodal distribution for OF8-2mer and 3HT-2mer with the peaks centred at approximately $\bar{\mu} \pm \mu_{\sigma}$. As the oligomer length increases we once again observe a better agreement to a normal unimodal data distribution. When we compare the single molecule approach to the ensemble averaged data we observe large differences ranging between $0.912 \mathrm{D}$ and 2.416 D for OF8-8mer and 3HT-8mer

respectively (See Table 1) The angle between the end-to-end length vector and the dipole moment $\left(\Theta^{D}\right)$ is provided in Fig. S8. For the OF8 and 3HT-2mer ensembles the majority of the points are condensed into a range between 60 to $120^{\circ}$, however, for the 8 mer ensembles we find a wider spread of points between 0 and $180^{\circ}$.

We finally turn our attention to the convergence of the moving average for the various systems. As per Fig. 10 we do not observe a clear convergence to $\bar{\mu}$ for any of the OF8 oligomers. However, for 3HT we observe a convergence by 1000 configurations for all oligomer lengths. When we look at the increased tolerance range we observe that the $3 \mathrm{HT}-2 \mathrm{mer}$ and 3HT-8mer are within these limits for all moving averages outwith the initial fluctuation. For 3HT-4mer we find this at a slight larger configuration amount of 300 configurations. For OF8-2mer we observe large fluctuations and finally residing in this range at approximately 800 configurations, which was also the case for the 4mer, occurring at 900 configurations. In contrast the OF8-8mer only requires as little as 250 configurations to remain within this range.

\section{Conclusion}

The configurational landscape of organic $\pi$-conjugated materials that have interest as active materials within organic photovoltaic devices were explored via molecular dynamics simulations. These ensembles were generated using a recently parameterised force-field ${ }^{20}$ specifically for organic $\pi$-conjugated materials. The resulting electronic structure of each 


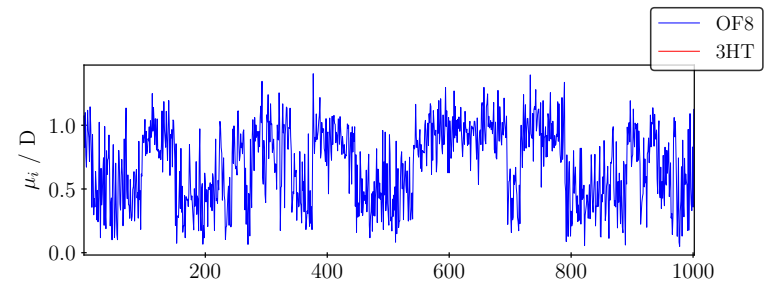

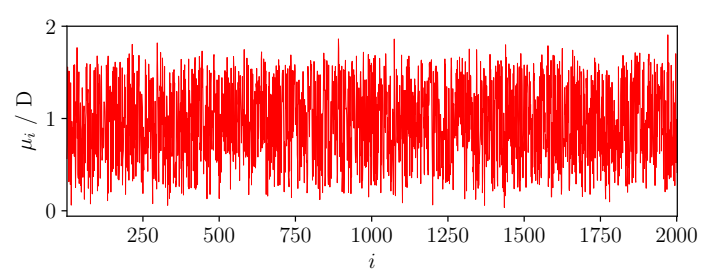

(a)

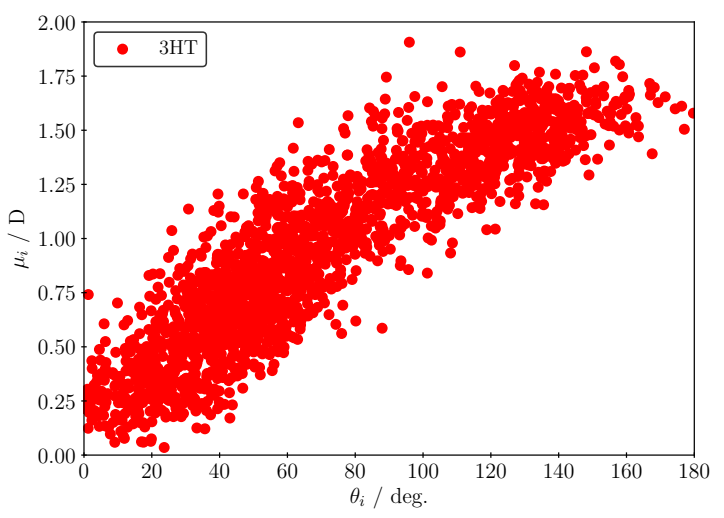

(c)

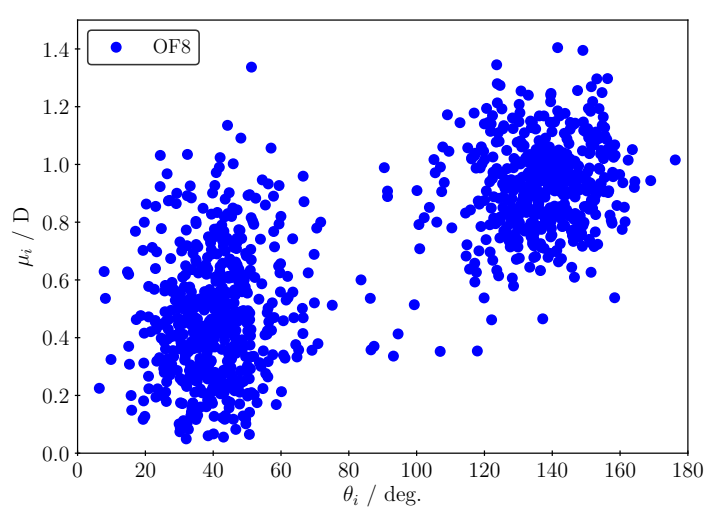

(b)

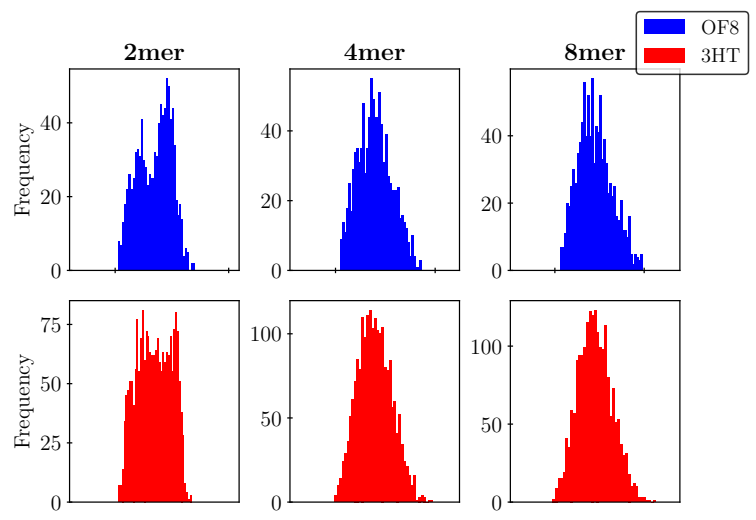

(d)

Figure 9: (a) The magnitude of the dipole moment for each individual OF8 and 3HT-2mer molecule within the simulated trajectory. (b) The magnitude of the dipole moment as a function of the intermolecular dihedral for each individual OF8-2mer molecule within the simulated trajectory. (c) The magnitude of the dipole moment as a function of the intermolecular dihedral for each individual 3HT-2mer molecule within the simulated trajectory. (d) Histogram representation of magnitude of the dipole moment for the ensembles considered, the abscissa of the histogram spans $\pm 5 \mu_{\sigma}$ from $\bar{\mu}$. 

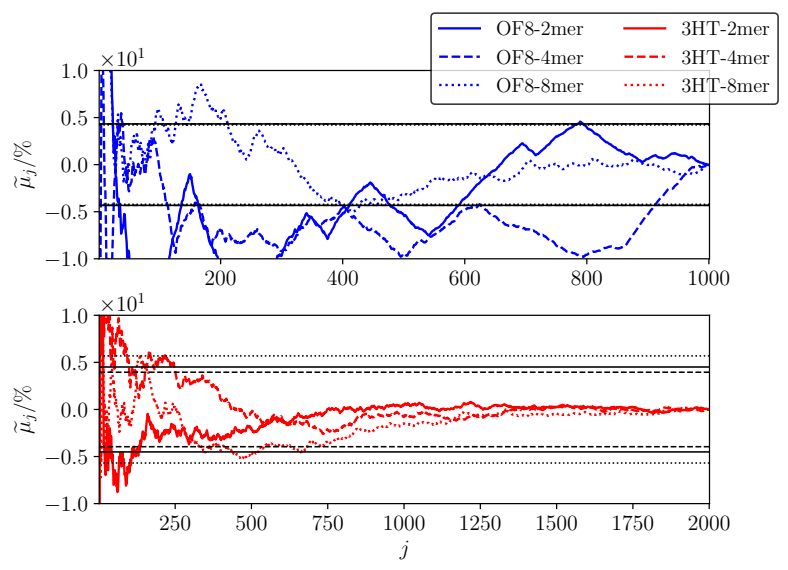

Figure 10: Convergence of $\widetilde{\mu}_{j}$ for each successive point across the simulated trajectory. The solid lines represent the quantity which would represent a deviation of $\pm \frac{1}{10} \mu_{\sigma}$ from $\bar{\mu}$.

configuration within the ensemble was investigated through the use of quantum chemical computations. This tandem approach allows one to explore the electronic properties not only on a single molecule but through the entire ensemble and ultimately aid in the design of materials with greater performance. The ensemble statistics for various properties such as the ground state electronic energy, ionisation potential, excitation energy to the first excited state and oscillator strength, and the magnitude of the dipole moment were explored. For all energetic based properties we find that the data sets reach a clear convergence at around 800 and 1000 configurations for OF8 and 3HT oligomers respectively. It was also observed for these properties that approximated ensemble averaged properties, deviating with respect to the true average by $\pm 10 \sigma_{x}$, could be attained using far reduced subsets, in some instances as little as 200 configurations when compared to the 1001 and 2001 configurations in the overall ensembles. For the oscillator strength the convergence is not as clear. In terms of OF8 we only observe convergence for the 2 mer and 8mer at approximately 900 configurations. For $3 \mathrm{HT}$ we observe clear convergence for the 2 mer and 8 mer at far larger configurational amounts than the aforementioned energetic properties. The increased tolerance range can once again be reached with far less configurations, with the exception of 3HT-8mer which required 1700 configurations. For the magnitude of the dipole moment the convergence is once again not as clear cut for the OF8, however, this is not true for the $3 \mathrm{HT}$ oligomers 
which all show a convergence at around the mid point of our simulations. In terms of the increased tolerance we observe that for $3 \mathrm{HT}$ oligomers this can be reached by approximately 300 configurations, however, this can only be achieved by OF8-8mer, with the smaller OF8 oligomers requiring upwards of 800 configurations.

\section{Supporting Information}

The Supporting Information is available free of charge at XX. The $x y z$ coordinates of each configuration within the sampled molecular dynamics ensembles are provided for each system of interest.

\section{Acknowledgement}

I.G., M.J.P., and J.W. acknowledge funding from EPSRC (awards EP/J009318/1 and EP/G03673X/1) and the Scottish Doctoral Training Centre in Condensed Matter Physics (CMCDT). M.J.P. also thanks the EPSRC for funding through Platform Grant EP/P001459/1 and Programme Grant EP/T021675. The authors also thank Dr Jeremy Coe for maintaining the high performance computing resources used within this work.

\section{References}

(1) Sprick, R. S.; Wilbraham, L.; Bai, Y.; Guiglion, P.; Monti, A.; Clowes, R.; Cooper, A. I.; Zwijnenburg, M. A. Nitrogen Containing Linear Poly(phenylene) Derivatives for Photocatalytic Hydrogen Evolution from Water. Chemistry of Materials 2018, 30, 5733-5742.

(2) Bai, Y.; Woods, D. J.; Wilbraham, L.; Aitchison, C. M.; Zwijnenburg, M. A.; Sprick, R. S.; Cooper, A. I. Hydrogen evolution from water using heteroatom substituted fluorene conjugated co-polymers. J. Mater. Chem. A 2020, 8, 8700-8705. 
(3) Zhu, L.; Wang, M.; Li, B.; Jiang, C.; Li, Q. High efficiency organic photovoltaic devices based on isoindigo conjugated polymers with a thieno[3,2-b]thiophene $\pi$-bridge. J. Mater. Chem. A 2016, 4, 16064-16072.

(4) Forrest, S. R. The path to ubiquitous and low-cost organic electronic appliances on plastic. Nature 2004, 428, 911-918.

(5) Guo, X.; Baumgarten, M.; Müllen, K. Designing $\pi$-conjugated polymers for organic electronics. Progress in Polymer Science 2013, 38, 1832 - 1908, Topical issue on Conductive Polymers.

(6) Abdulrazzaq, O. A.; Saini, V.; Bourdo, S.; Dervishi, E.; Biris, A. S. Organic Solar Cells: A Review of Materials, Limitations, and Possibilities for Improvement. Particulate Science and Technology 2013, 31, 427-442.

(7) Khan, N.; Kausar, A.; Rahman, A. U. Modern Drifts in Conjugated Polymers and Nanocomposites for Organic Solar Cells: A Review. Polymer-Plastics Technology and Engineering 2015, 54, 140-154.

(8) Sekine, C.; Tsubata, Y.; Yamada, T.; Kitano, M.; Doi, S. Recent progress of high performance polymer OLED and OPV materials for organic printed electronics. Science and Technology of Advanced Materials 2014, 15, 034203, PMID: 27877671.

(9) Solanki, C. Solar Photovoltaics: Fundamentals, Technologies And Applications; PHI Learning, 2015.

(10) Facchetti, A. Polymer donor-polymer acceptor (all-polymer) solar cells. Materials Today 2013, 16, $123-132$.

(11) Liao, S.-H.; Jhuo, H.-J.; Cheng, Y.-S.; Chen, S.-A. Fullerene Derivative-Doped Zinc Oxide Nanofilm as the Cathode of Inverted Polymer Solar Cells with Low-Bandgap Polymer (PTB7-Th) for High Performance. Advanced Materials 2013, 25, 4766-4771. 
(12) Kippelen, B.; Brédas, J.-L. Organic photovoltaics. Energy $\&$ Environmental Science 2009, 2, 251-261.

(13) Coropceanu, V.; Cornil, J.; da Silva Filho, D. A.; Olivier, Y.; Silbey, R.; Brédas, J.-L. Charge Transport in Organic Semiconductors. Chemical Reviews 2007, 107, 926-952.

(14) de Hatten, X.; Cournia, Z.; Huc, I.; Smith, J.; Metzler-Nolte, N. Force-Field Development and Molecular Dynamics Simulations of Ferrocene-Peptide Conjugates as a Scaffold for Hydrogenase Mimics. Chemistry - A European Journal 2007, 13, 81398152.

(15) Lopes, P. E. M.; Guvench, O.; MacKerell, A. D. In Molecular Modeling of Proteins; Kukol, A., Ed.; Springer New York: New York, NY, 2015; pp 47-71.

(16) Robustelli, P.; Piana, S.; Shaw, D. E. Developing a molecular dynamics force field for both folded and disordered protein states. Proceedings of the National Academy of Sciences 2018, 115, E4758-E4766.

(17) Moreno, M.; Casalegno, M.; Raos, G.; Meille, S. V.; Po, R. Molecular Modeling of Crystalline Alkylthiophene Oligomers and Polymers. The Journal of Physical Chemistry B 2010, 114, 1591-1602, PMID: 20058895.

(18) Bhatta, R. S.; Yimer, Y. Y.; Perry, D. S.; Tsige, M. Improved Force Field for Molecular Modeling of Poly(3-hexylthiophene). The Journal of Physical Chemistry B 2013, 117, 10035-10045, PMID: 23899343.

(19) DuBay, K. H.; Hall, M. L.; Hughes, T. F.; Wu, C.; Reichman, D. R.; Friesner, R. A. Accurate Force Field Development for Modeling Conjugated Polymers. Journal of Chemical Theory and Computation 2012, 8, 4556-4569, PMID: 26605615.

(20) Wildman, J.; Repiscak, P.; Paterson, M. J.; Galbraith, I. General Force-Field 
Parametrization Scheme for Molecular Dynamics Simulations of Conjugated Materials in Solution. Journal of Chemical Theory and Computation 2016, 12, 3813-3824.

(21) Jorgensen, W. L.; Maxwell, D. S.; Tirado-Rives, J. Development and Testing of the OPLS All-Atom Force Field on Conformational Energetics and Properties of Organic Liquids. Journal of the American Chemical Society 1996, 118, 11225-11236.

(22) Jorgensen, W. L.; McDonald, N. A. Development of an all-atom force field for heterocycles. Properties of liquid pyridine and diazenes. Journal of Molecular Structure: THEOCHEM 1998, 424, 145 - 155, A Faithful Couple: Qualitative and Quantitative Understanding of Chemistry.

(23) McDonald, N. A.; Jorgensen, W. L. Development of an All-Atom Force Field for Heterocycles. Properties of Liquid Pyrrole, Furan, Diazoles, and Oxazoles. The Journal of Physical Chemistry B 1998, 102, 8049-8059.

(24) Rizzo, R. C.; Jorgensen, W. L. Journal of the American Chemical Society 1999, 121, $4827-4836$.

(25) Price, M. L. P.; Ostrovsky, D.; Jorgensen, W. L. Gas-phase and liquid-state properties of esters, nitriles, and nitro compounds with the OPLS-AA force field. Journal of Computational Chemistry 2001, 22, 1340-1352.

(26) Watkins, E. K.; Jorgensen, W. L. Perfluoroalkanes: Conformational Analysis and Liquid-State Properties from ab Initio and Monte Carlo Calculations. The Journal of Physical Chemistry A 2001, 105, 4118-4125.

(27) Kaminski, G. A.; Friesner, R. A.; Tirado-Rives, J.; Jorgensen, W. L. Evaluation and Reparametrization of the OPLS-AA Force Field for Proteins via Comparison with Accurate Quantum Chemical Calculations on Peptides. The Journal of Physical Chemistry B 2001, 105, 6474-6487. 
(28) Yanai, T.; Tew, D. P.; Handy, N. C. A new hybrid exchange-correlation functional using the Coulomb-attenuating method (CAM-B3LYP). Chemical Physics Letters 2004, 393, $51-57$.

(29) Bloom, J. W. G.; Wheeler, S. E. Benchmark Torsional Potentials of Building Blocks for Conjugated Materials: Bifuran, Bithiophene, and Biselenophene. Journal of Chemical Theory and Computation 2014, 10, 3647-3655, PMID: 26588510.

(30) Aschi, M.; Amadei, A.; Pellegrino, A.; Perin, N.; Po, R. Thermal and environmental effects on Oligothiophene low-energy singlet electronic excitations in dilute solution: a theoretical and experimental study. Theoretical Chemistry Accounts 2012, 131, 1-14.

(31) Bockmann, M.; Schemme, T.; de Jong, D. H.; Denz, C.; Heuer, A.; Doltsinis, N. L. Structure of P3HT crystals, thin films, and solutions by UV/Vis spectral analysis. Phys. Chem. Chem. Phys. 2015, 17, 28616-28625.

(32) Spoel, D. V. D.; Lindahl, E.; Hess, B.; Groenhof, G.; Mark, A. E.; Berendsen, H. J. C. GROMACS: Fast, flexible, and free. Journal of Computational Chemistry 2005, 26, $1701-1718$.

(33) Hess, B.; Kutzner, C.; Spoel, D. V. D.; Lindahl, E. Gromacs 4: algorithms for highly efficient, load-balanced, and scalable molecular simulation. Journal of Chemical Theory and Computation 2008, 4, 435-447.

(34) Yaws, C. Yaws' handbook of thermodynamic and physical properties of chemical compounds: physical, thermodynamic and transport properties for 5,000 organic chemical compounds; Knovel, 2003.

(35) Inoue, S.; Minemawari, H.; Tsutsumi, J.; Chikamatsu, M.; Yamada, T.; Horiuchi, S.; Tanaka, M.; Kumai, R.; Yoneya, M.; Hasegawa, T. Effects of Substituted Alkyl Chain Length on Solution-Processable Layered Organic Semiconductor Crystals. Chemistry of Materials 2015, 27, 3809-3812. 
(36) Frisch, M. J.; Trucks, G. W.; Schlegel, H. B.; Scuseria, G. E.; Robb, M. A.; Cheeseman, J. R.; Scalmani, G.; Barone, V.; Petersson, G. A.; Nakatsuji, H. et al. Gaussian 09 Revision D.01. 2016; Gaussian Inc.: Wallingford CT.

(37) Frisch, M. J.; Trucks, G. W.; Schlegel, H. B.; Scuseria, G. E.; Robb, M. A.; Cheeseman, J. R.; Scalmani, G.; Barone, V.; Petersson, G. A.; Nakatsuji, H. et al. Gaussian 16 Revision A.03. 2016; Gaussian Inc.: Wallingford CT.

(38) Hohenberg, P.; Kohn, W. Inhomogeneous Electron Gas. Phys. Rev. 1964, 136, B864B871.

(39) Kohn, W.; Sham, L. J. Self-Consistent Equations Including Exchange and Correlation Effects. Phys. Rev. 1965, 140, A1133-A1138.

(40) Runge, E.; Gross, E. K. U. Density-Functional Theory for Time-Dependent Systems. Physical Review Letters 1984, 52, 997-1000.

(41) Virtanen, P.; Gommers, R.; Oliphant, T. E.; Haberland, M.; Reddy, T.; Cournapeau, D.; Burovski, E.; Peterson, P.; Weckesser, W.; Bright, J. et al. SciPy 1.0: Fundamental Algorithms for Scientific Computing in Python. Nature Methods 2020, 17, 261-272. 